\title{
Microstructure, Mechanical, and Biological Properties of Porous Poly(vinylidene fluoride) Scaffolds Fabricated by Selective Laser Sintering
}

\author{
Wei Huang, 1,2 Pei Feng, ${ }^{2}$ Chengde Gao, ${ }^{2}$ Xiong Shuai, ${ }^{3}$ Tao Xiao, \\ Cijun Shuai, ${ }^{2,4}$ and Shuping Peng ${ }^{1,6}$ \\ ${ }^{1}$ Hunan Provincial Tumor Hospital and the Affiliated Tumor Hospital of Xiangya School of Medicine, \\ Central South University, Changsha 410013, China \\ ${ }^{2}$ State Key Laboratory of High Performance Complex Manufacturing, Central South University, Changsha 410083, China \\ ${ }^{3}$ State Key Laboratory of Powder Metallurgy, Central South University, Changsha 410083, China \\ ${ }^{4}$ Orthopedic Biomedical Materials Institute, Central South University, Changsha 410083, China \\ ${ }^{5}$ Department of Orthopedics, The Second Xiangya Hospital, Central South University, Changsha 410011, China \\ ${ }^{6}$ School of Basic Medical Science, Central South University, Changsha 410078, China
}

Correspondence should be addressed to Cijun Shuai; shuai@csu.edu.cn and Shuping Peng; shuping@csu.edu.cn

Received 26 January 2015; Revised 23 April 2015; Accepted 27 April 2015

Academic Editor: Piotr Wilczek

Copyright ( $\odot 2015$ Wei Huang et al. This is an open access article distributed under the Creative Commons Attribution License, which permits unrestricted use, distribution, and reproduction in any medium, provided the original work is properly cited.

Porous poly(vinylidene fluoride) (PVDF) scaffolds were prepared by selective laser sintering. The effects of laser energy density, ranging from 0.66 to $2.16 \mathrm{~J} / \mathrm{mm}^{2}$, on microstructure and mechanical properties were investigated. At low energy density levels, PVDF particles could fuse well and the structure becomes dense with the increase of the energy density. Smoke and defects (such as holes) were observed when the energy density increased above $1.56 \mathrm{~J} / \mathrm{mm}^{2}$ which indicated decomposition of the PVDF powder. The scaffolds appeared to be light yellow and there was a reduction in tensile strength. The fabricated scaffolds were immersed into simulated body fluid for different time to evaluate biostability. In addition, MG63 cells were seeded and cultured for different days on the scaffolds. The testing results showed that the cells grew and spread well, indicating that PVDF scaffolds had good biocompatibility.

\section{Introduction}

Autografts were the gold standard for bone transplantation, but their existing problems, for example, finite availability and donor site complication, had limited their clinical application [1-3]. Hence artificial bone had been used for bone regeneration. Tridimensional porous scaffolds played a crux role in synthetic bone, because the scaffolds could imitate the bone constructions and role of the extracellular matrix and endorse cell conglutination, multiplication, and differentiation. Besides they could transport nutrient and metabolic waste [4-7].

Scaffolds were fabricated by some conventional methods which mainly contained solvent casting, injection molding, and electrospinning $[8,9]$. However, these methods required a separate mold for exterior geometry and could not control over the porous architecture precisely [10]. Solid freeform fabrication (SFF) methods could precisely control over the objects external shape and internal pore interconnectivity [11-16]. Selective laser sintering (SLS), as a family member of the SFF methods, employed a laser to selectively sinter thin layers of powder materials to form solid 3D objects [17, 18].

Poly(vinylidene fluoride) (PVDF) was a biocompatibility polymer. Zhang et al. [19] proved that PVDF possessed hemocompatibility and prepared PVDF hollow fiber hemodialysis membranes. Agyemang et al. [20] had found that PVDF-calcium phosphate composite scaffolds had potential applications in tissue engineering. Guo et al. [21] used 
TABLE 1: The SLS process parameters.

\begin{tabular}{lc}
\hline Parameters & Value \\
\hline Spot diameter $(\mathrm{mm})$ & 0.8 \\
Scan space $(\mathrm{mm})$ & 3.0 \\
Layer thickness $(\mathrm{mm})$ & $0.1 \sim 0.2$ \\
\hline
\end{tabular}

polyurethane/polyvinylidene fluoride scaffolds for trauma recovery applications.

In this study, PVDF scaffolds were fabricated via SLS for bone regeneration, and the effects of the laser energy density on microstructure and mechanical performance were studied with scanning electron microscopy (SEM), a universal testing system, respectively. The biostability was evaluated by immersing the scaffolds into SBF. Besides, MG63 cells were planted on PVDF scaffolds and in vitro cell action, containing cell attachment and multiplication, was researched.

\section{Materials and Methods}

2.1. Material. PVDF powder $\left(M_{w}=275,000 \mathrm{~g} / \mathrm{mol}\right)$ was purchased from Huangjiang Huayi Plastics Material Co., Guang Dong, China. Density was $1.75-1.78 \mathrm{~g} / \mathrm{cm}^{3}$. It had a melting point of $170^{\circ} \mathrm{C}$ and the long-term use temperature of $-40 \sim 150^{\circ} \mathrm{C}$, and the crystallinity index was approximately $50 \%$.

2.2. Fabrication. Scaffolds were fabricated using a homemade SLS system which has been reported previously [22, 23]. When a process was developed for fabricating synthetic bone scaffolds, process parameters should be suitable. An important factor to the success of the sintering process was the laser energy density (ED). It was defined by

$$
\mathrm{ED}=\frac{P}{V \times D}\left(\mathrm{~J} / \mathrm{mm}^{2}\right)
$$

where the laser energy density is ED, the laser power is $P$, the scan speed is $V$, and the spot diameter is $D$ [24]. The sintered process parameters were tabulated in Table 1.

2.3. Characterization. The morphologies of the scaffolds were observed with a SEM (JEOL Co., Japan). The technical parameters of SEM were that the resolution was $\alpha<$ $3.0 \mathrm{~nm}$ and magnification times was 20 300,000. Before SEM observation, the scaffolds were platinum coated with a sputter coater (JEOL Co., Japan). The scaffolds were analyzed by Xray diffraction (XRD) (Bruker AXS Inc., Germany) with the Cu target, $\lambda=1.54056 \AA$, and $\mathrm{K}_{\alpha} \mathrm{X}$-ray. $2 \theta$ of XRD graph was registered in the space $\left(10^{\circ}-70^{\circ}\right)$ at the rate of $2^{\circ} / \mathrm{min}$.

Tensile strength of scaffolds was measured by using a universal mechanical tester (Shanghai Zhuoji Instruments Co. Ltd., China) at environment temperature. The speed of crosshead was selected at $5 \mathrm{~mm} / \mathrm{min}$ and the stress-strain curve was recorded during the tests. Five specimens were tested for each set of process conditions.
2.4. Immersion in SBF. The scaffolds were immersed into simulated body fluid (SBF) to evaluate their in vitro biostability. Ion concentrations in SBF were close to the inorganic fraction of blood drug concentration. The SBF solution was obtained by dissolving $\mathrm{KH}_{2} \mathrm{PO}_{4}, \mathrm{NaHCO}_{3}, \mathrm{NaCl}$, $\mathrm{MgSO}_{4} \cdot 7 \mathrm{H}_{2} \mathrm{O}, \mathrm{CaCl}_{2}$, and $\mathrm{KCl}$ into aqua destillata and buffering with Tris-HCL to $\mathrm{pH} 7.4$ at human body temperature $\left(37^{\circ} \mathrm{C}\right)$ [25]. The scaffolds were soaked in SBF for $1,4,7$, and 14 days at human body temperature $\left(37^{\circ} \mathrm{C}\right)$. They, after removal from the fluid, were rinsed with deionized water and dried in cryogenic nitrogen airflow and then weighed. The weight remaining percentage was calculated by

$$
\text { Weight remaining } \%=100-\frac{W_{0}-W_{t}}{W_{0}} \times 100 \text {, }
$$

where the initial dry weight is $W_{0}$ and the weight after immersing in SBF is $W_{t}$.

2.5. Cell Culture. To evaluate the biocompatibility of the scaffolds, a cell culture research was carried out. Before the experiment, the scaffolds were sterilized. In detail the process was described as follows: the samples were immersed in $70 \%$ ethyl alcohol solution $2 \mathrm{~h}$ and afterwards rinsed twice in phosphate buffered saline to shift the ethyl alcohol, followed by exposure to ultraviolet light $15 \mathrm{~min}$ for each side.

MG63 osteoblast-like cells were used for the assessment of the biocompatibility of the scaffolds. The cells were fostered in Dulbecco's modified Eagle's medium (DMEM, HyClone, USA) under normal situation. Besides, $10 \%$ fetal bovine serum and gentamicin were added into DMEM. Approximately $50 \mu \mathrm{L}$ of culture medium containing $5 \times 10^{4}$ cells was planted on the scaffolds and cultured for 1, 3, and 5 days in condition of $5 \% \mathrm{CO}_{2}$ at human body temperature $\left(37^{\circ} \mathrm{C}\right)$. The solution was changed every second day.

2.5.1. SEM Techniques. The cell morphologies on the scaffold were investigated using SEM. After the preselected fostering days, the scaffolds were rinsed with phosphate buffer saline (PBS) two times following settlement with $4 \%$ formalin in PBS ( $\mathrm{pH}$ 7.4). Finally, the scaffolds were rinsed with PBS two times and dewatered in a gradient ethanol series from 50 to $100 \%$. The scaffolds were air drying in a dryer overnight. They were then coated with platinum for SEM observation.

2.5.2. Fluorescence Techniques. Fluorescence technique was used to analyse cell-material interaction. After the cell culture experiment, cells were cleaned with PBS, settled with paraformaldehyde (Sigma, St. Louis, MI, USA), washed, and then permeabilized with $0.1 \%$ Tween 20 . Afterwards, the cell was rinsed with PBS and then preincubated by $1 \%$ gelatin in PBS 20 minute prior to incubation in a compound of $4 \mu \mathrm{M}$ EthD-1 and $2 \mu \mathrm{M}$ calcein AM in PBS for $30 \mathrm{~min}$ in a humid chemostat. Fluorescence figures were achieved straightway with a confocal microscope (Leica Microsystem, Mannheim, Germany).

2.6. Statistical Analysis. The experimental data were analyzed by origin 8.0 software and the results were depicted as 


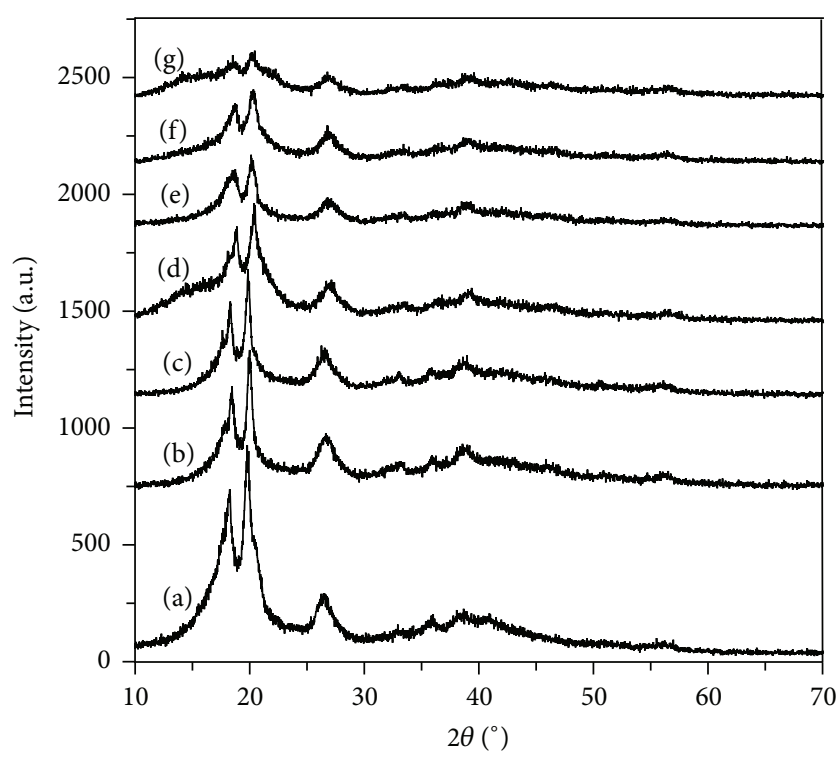

FIGURE 1: XRD patterns of PVDF powder (a) and PVDF scaffolds sintered at different laser energy density of (b) 0.66 ; (c) 0.96 ; (d) 1.26 ; (e) 1.56 ; (f) 1.86 ; (g) $2.16 \mathrm{~J} / \mathrm{mm}^{2}$.

mean \pm standard deviation. Data were assessed for statistical significance using Student's $t$-test; statistical significance was selected at $P<0.05$.

\section{Results and Discussions}

3.1. Composition Analysis. XRD spectrums for PVDF powder and PVDF scaffolds sintered at different energy density were shown in Figure 1. It could be seen that there were three distinctive peaks occurring at $2 \theta$ angle of $18.3^{\circ}, 20.0^{\circ}$, and $26.6^{\circ}$. These results accorded with the value of $2 \theta$ for the standard in the literature [26]. The peaks intensities decreased with increasing the energy density. This might be because the crystallinity rate was associated with symmetry and flexibility of the main molecular chain. In order to achieve the requirement for sintering, the sintering temperature was higher than the melting temperature $\left(T_{m}\right)$. Therefore, the molecular chain was destroyed during the sintering process. In the meantime, the change process of material from melt to solidification was fast. Therefore the crystallization time was short, which leaded to the decrease of the crystallinity degree.

3.2. Morphologies. The surface morphologies of the scaffolds built with different laser ED were shown in Figure 2. At a low ED of $0.66 \mathrm{~J} / \mathrm{mm}^{2}$ (Figure 2(a)), particles did not melt completely. They could be identified, and a number of pores with irregular shape were seen on the top surface. The surface morphology did not generally change except for the increase fusion of particles when the ED increased to $0.96 \mathrm{~J} / \mathrm{mm}^{2}$ (Figure 2(b)). As observed in Figure 2(c), the surface morphology changed greatly for the energy density up to $1.26 \mathrm{~J} / \mathrm{mm}^{2}$, and the scaffold surface became flat and smooth, but some small holes remained on the surface. In Figure 2(d), the surface became dense, and the holes could hardly be found. It could be explained that the better fusion facilitated the flow of the material. However, some defects such as holes appeared when the ED was ulteriorly improved (Figures 2(e)-2(f)). Furthermore, the holes get more and big with increasing the energy density.

The fractured surfaces of the scaffolds which were sintered at different energy density were shown in Figure 3. There was a clear layer structure in Figures 3(a)-3(b). As the ED improved to $1.26 \mathrm{~J} / \mathrm{mm}^{2}$ (Figure $3(\mathrm{c})$ ), layer constructions remained, but the cross sections became dense which accorded with the development of a continuous surface as shown in Figure 2(c). Meanwhile, some irregular holes still remained. One possible reason could explain the phenomenon; the energy density was not sufficiently high to lead to the full melt of the entire layer. At energy density of $1.56 \mathrm{~J} / \mathrm{mm}^{2}$ (Figure 3(d)), layer structure disappeared and structure became dense. Meanwhile, the holes changed from irregular to spherical (Figures 3(c)-3(d)). It was due to a more complete fusion of polymer particles, which caused the irregular holes to turn into globose due to the influence of the surface tension of the fused polymer.

When the ED ulteriorly increased (Figures 3(e)-3(f)), the holes get big and the surface changed from smoothness to roughness. Furthermore, the color of scaffolds changed and smoke was generated, which indicated that thermal decomposition of the polymer material occurred. The smoke of layer surface escaped easily. Of course, the laser beam could penetrate through the skin layer and reach a deep section, so the thermal decomposition of the polymer in the internal might occur. In the decomposition process, if the formation of holes and generation of smoke were not synchronized, the smoke would be trapped. And the trapped smoke could cause the holes to become large. Another possible reason for the big holes in Figure 3(f) could be the merging of the holes.

3.3. Tensile Strength. Tensile strength of scaffolds sintered at different energy density was shown in Figure 4. The tensile strength increased with improving the ED. However, as the ED was above $1.56 \mathrm{~J} / \mathrm{mm}^{2}$, the tensile strength decreased. The optimum tensile strength was $45.31 \pm 0.52 \mathrm{MPa}$ at the energy density of $1.56 \mathrm{~J} / \mathrm{mm}^{2}$. The change trend of tensile strength accorded with the microstructural change of the scaffolds. The scaffolds with optimum mechanical properties were used in experiments of SBF and cell culture.

3.4. Scaffold Fabrication. The scaffold fabricated under the energy density of $1.56 \mathrm{~J} / \mathrm{mm}^{2}$ was shown in Figure 5 . The unsintered powder was removed by shaking the scaffolds, and then the residuary powder was cleaned by compressed gases. It could be seen that the scaffold had an integrated construction and fine stability. In addition, it showed a completely connected porous construction that is beneficial to the transmission of nutrients and the adhesion, proliferation, and differentiation of cells.

3.5. Biostability. The surface morphologies of the scaffolds immersed in SBF for 7 and 14 days were exhibited in Figure 6. It could be found that there was no obvious change in 


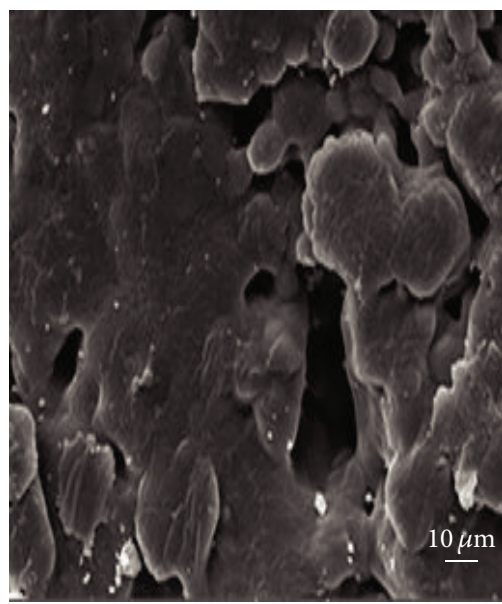

(a)

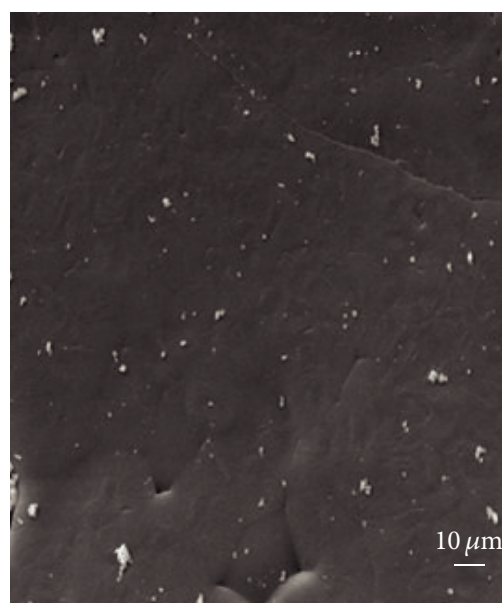

(d)

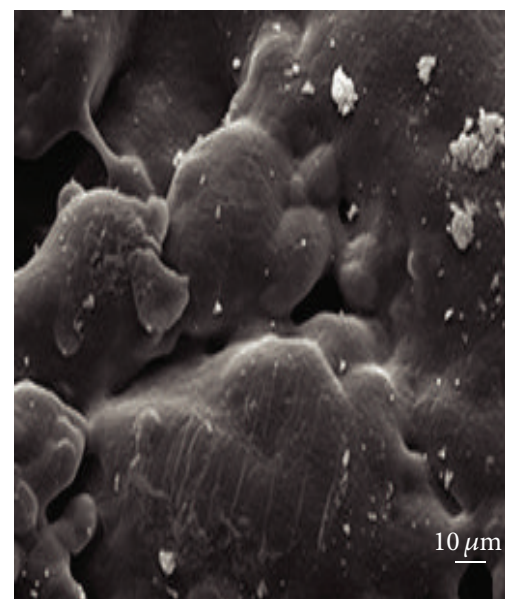

(b)

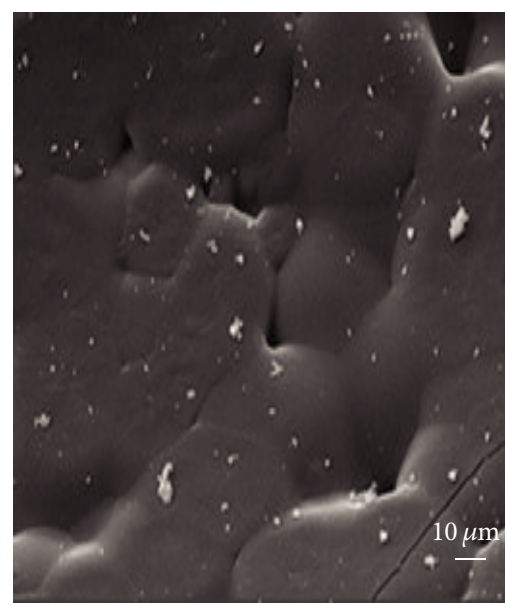

(e)

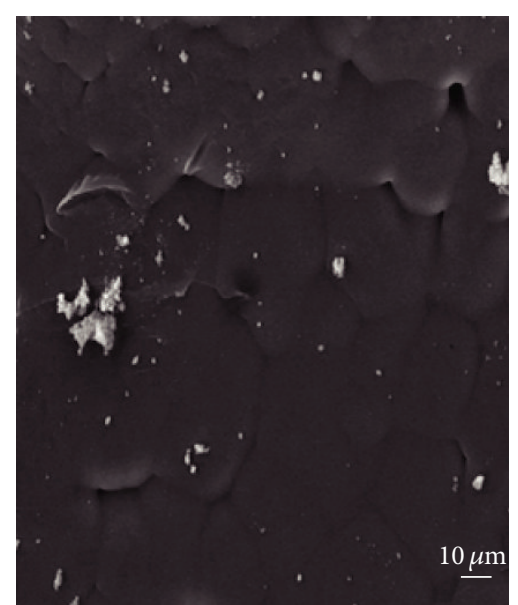

(c)

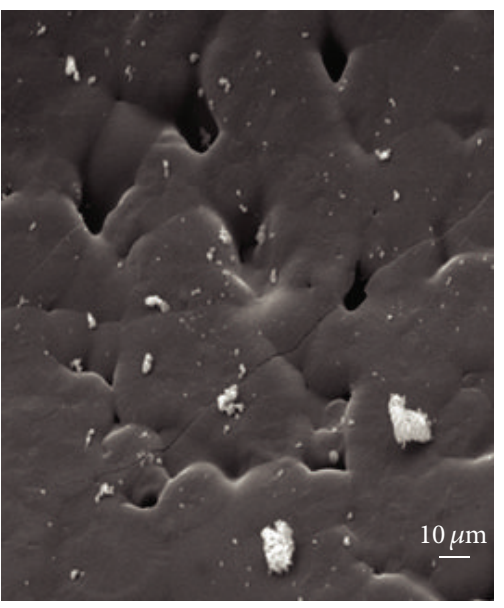

(f)

FIGURE 2: Surface of PVDF scaffolds sintered at energy density of (a) 0.66 ; (b) 0.96 ; (c) 1.26 ; (d) 1.56 ; (e) 1.86 ; (f) $2.16 \mathrm{~J} / \mathrm{mm}^{2}$.

shape and appearance of the scaffolds. The remaining weight percentage of PVDF scaffolds after immersing in SBF for different days was exhibited in Figure 7. It could be found that the weight of the scaffolds had small change during the test time. The results accorded with the variation of the morphology of the scaffolds exhibited in Figure 6.

3.6. Cell Culture. The morphologies of the MG63 cells after culturing for different days on the scaffolds were shown in Figure 8. At day 1 (Figure 8(a)), MG63 cells on the scaffolds showed a fusiform shape and anchored to the surface by filopodia. When the cells were cultured for 3 days, the cells grew and were connected together by their pseudopodia, which was exhibited in Figure 8(b). After fostering for 5 days, the MG63 cell presented a flat and spreading shape to form a confluent cell layer (Figure 8(c)). Fluorescence technique was used to analyse cell viability. Figure 9 showed fluorescence figures of MG63 cell fostered on the scaffolds after different days. It could be seen that viable cells were green, were attached fine, and exhibited ordinary growth on the scaffolds. The number of cells increased with the culture time from 1 to 5 days. The magnified images showed that cells grew and were connected together from 1 to 5 days which accorded with the development as shown in Figure 8. This observation indicated that PVDF scaffolds were suitable for MG63 cell proliferation. The SEM and fluorescence test results demonstrated that the PVDF scaffolds had good biocompatibility.

\section{Conclusions}

In this study, the PVDF scaffolds were successfully fabricated by SLS, and the microstructure, mechanical, and biological performance of the PVDF scaffolds were evaluated. The test consequence showed that the optimum sintering energy density was $1.56 \mathrm{~J} / \mathrm{mm}^{2}$. The tensile strength increased with increasing the ED from 0.66 to $1.56 \mathrm{~J} / \mathrm{mm}^{2}$ and decreased when the energy density exceeded $1.56 \mathrm{~J} / \mathrm{mm}^{2}$. The optimum tensile strength was $45.31 \pm 0.52 \mathrm{MPa}$. The SBF experiments indicated that PVDF scaffolds had good biostability. MG63 cells adhered well and grew on the PVDF scaffolds, which indicated good biocompatibility. The results indicated that 


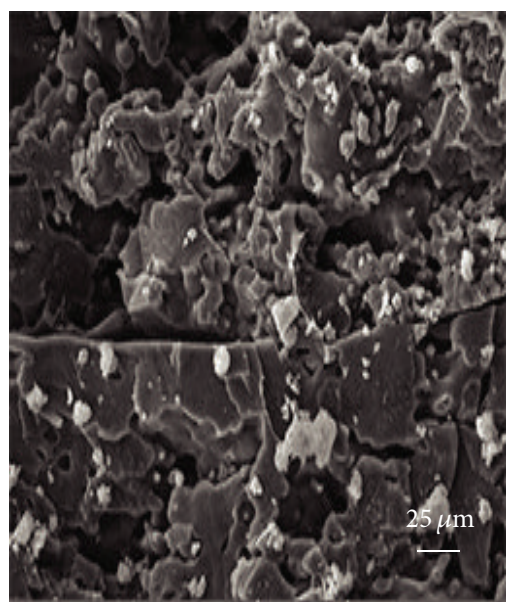

(a)

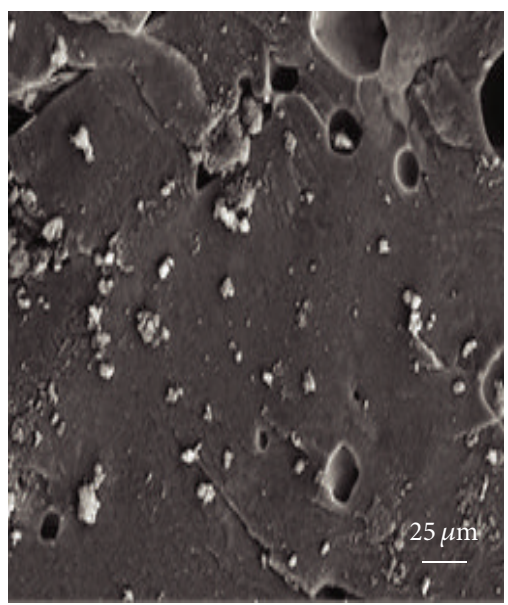

(d)

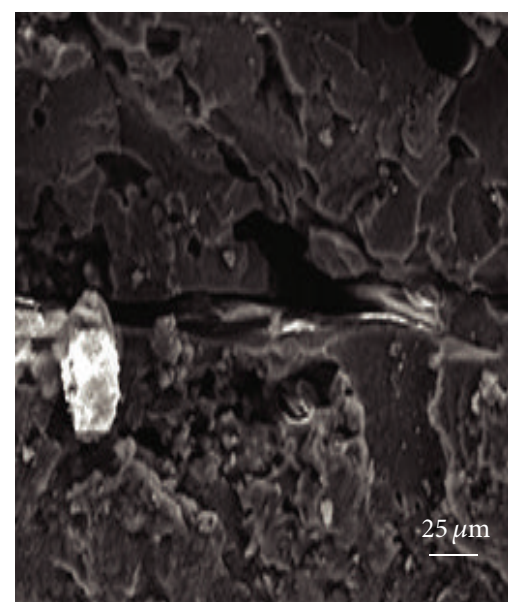

(b)

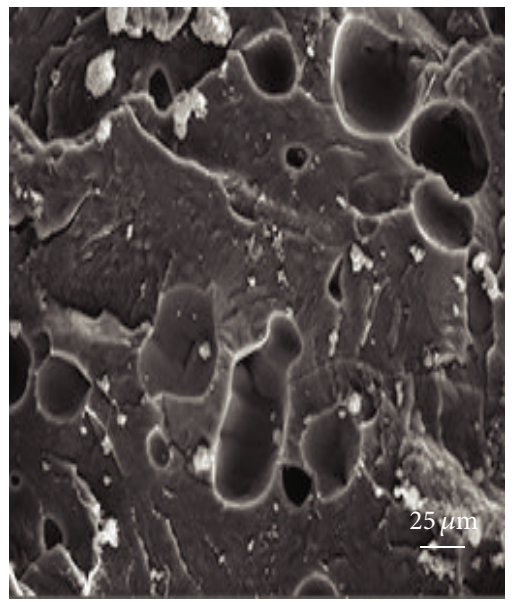

(e)

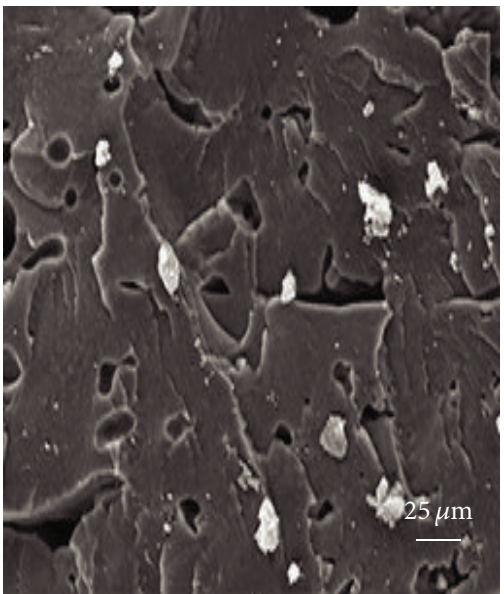

(c)

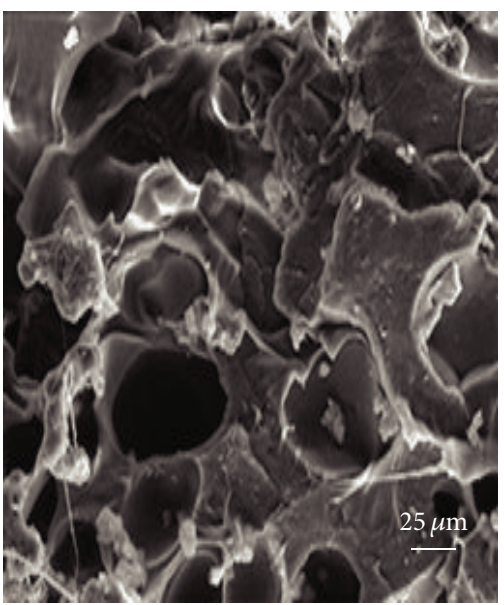

(f)

FigURE 3: Fractured cross sections of PVDF scaffolds sintered at different energy density of (a) 0.66; (b) 0.96; (c) 1.26; (d) 1.56; (e) 1.86; (f) $2.16 \mathrm{~J} / \mathrm{mm}^{2}$.

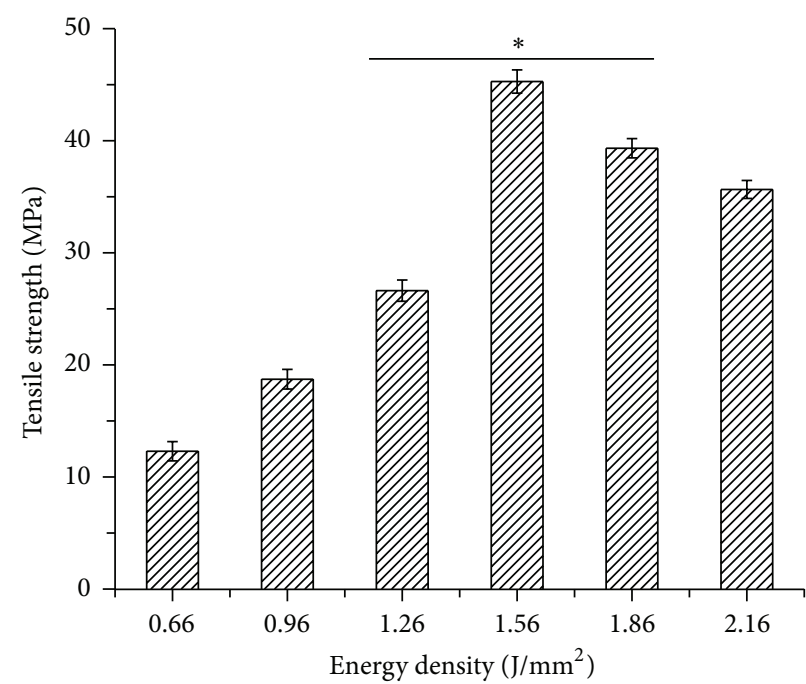

Figure 4: The relationship of tensile strength and energy density $\left({ }^{*} P<0.05\right)$. 


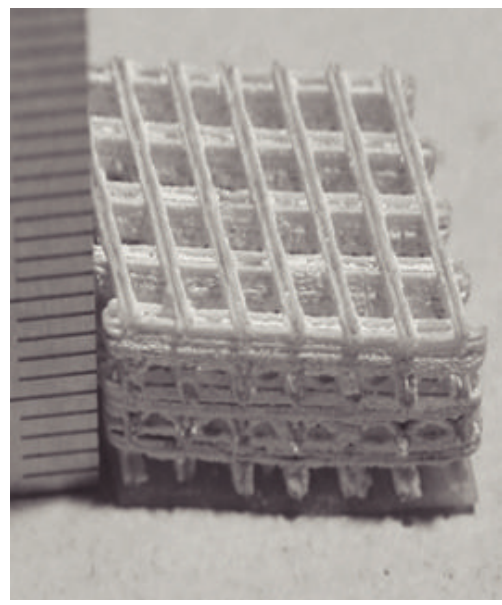

(a)

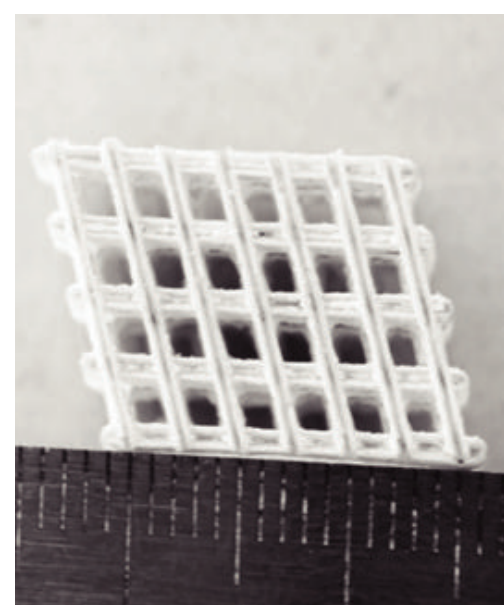

(b)

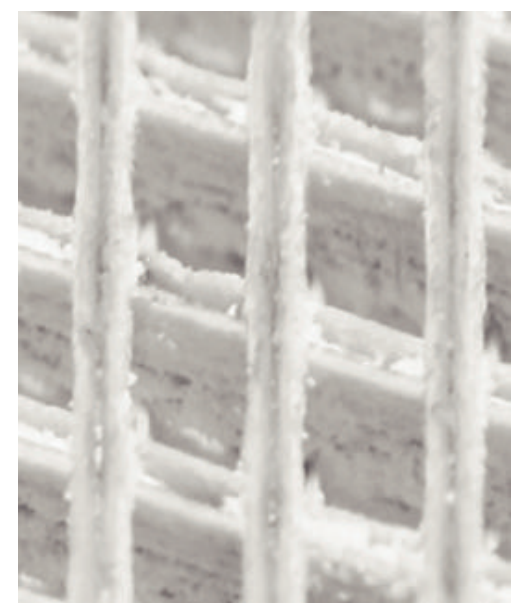

(c)

FIGURE 5: The interconnected porous scaffold fabricated by SLS: (a) isometric view; (b) top view; (c) magnifying view.

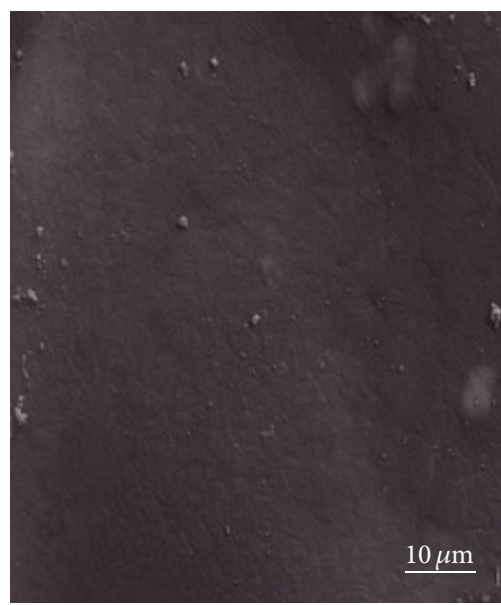

(a)

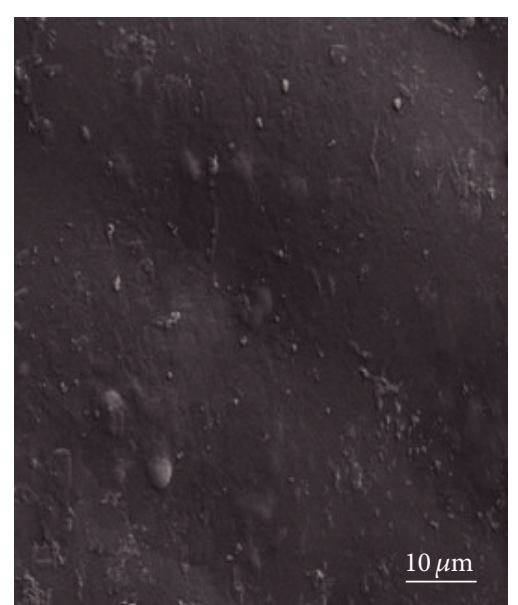

(b)

FIGURE 6: PVDF scaffolds immersed in SBF for different days: (a) 7 days; (b) 14 days.

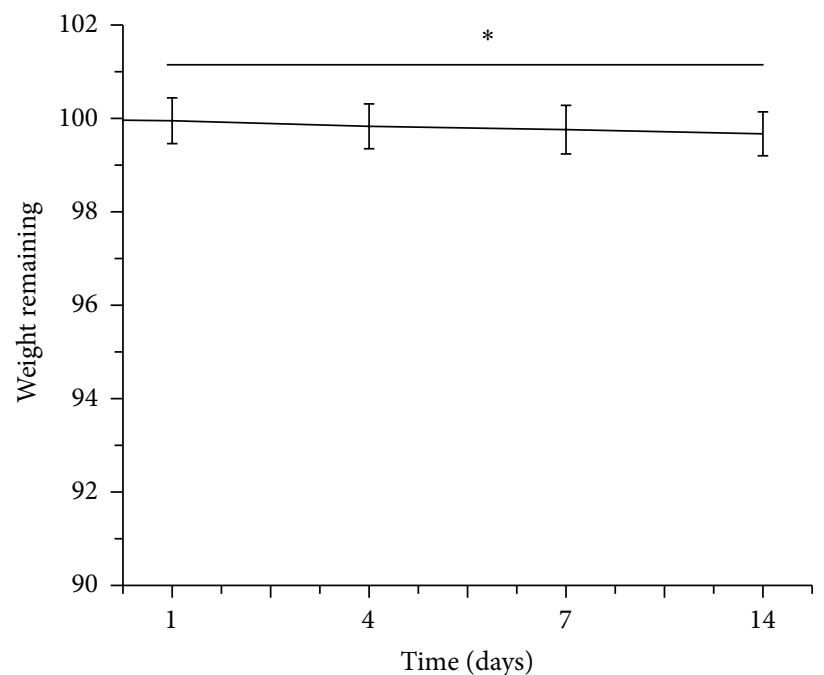

FIgURE 7: The remaining weight percentage of PVDF scaffolds after immersing in SBF for different days $\left({ }^{*} P<0.05\right)$. 


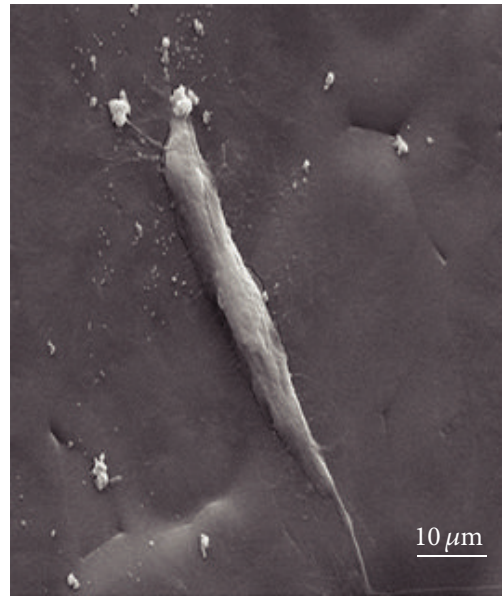

(a)

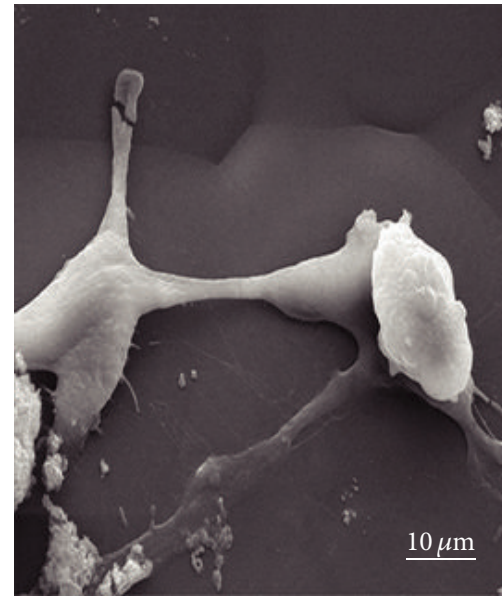

(b)

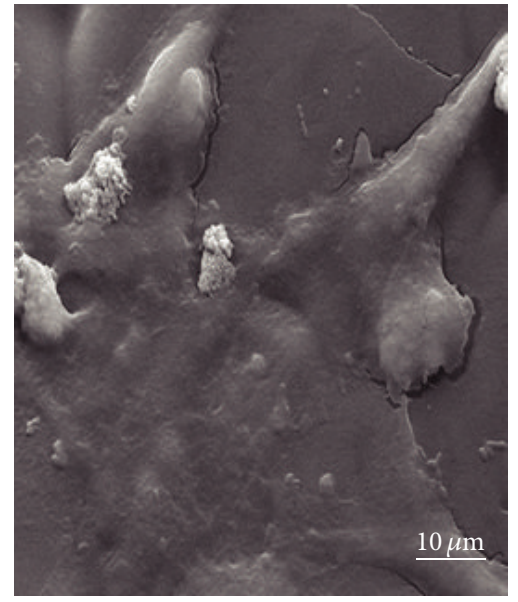

(c)

FIGURE 8: MG63 cells cultured on PVDF scaffolds for different days: (a) 1 day; (b) 3 days; (c) 5 days.

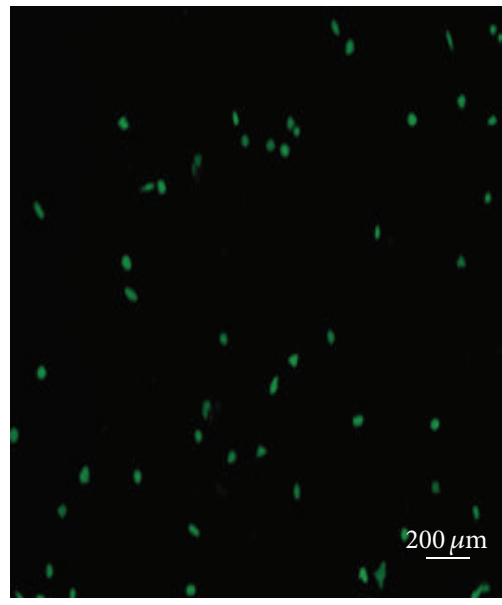

(a)

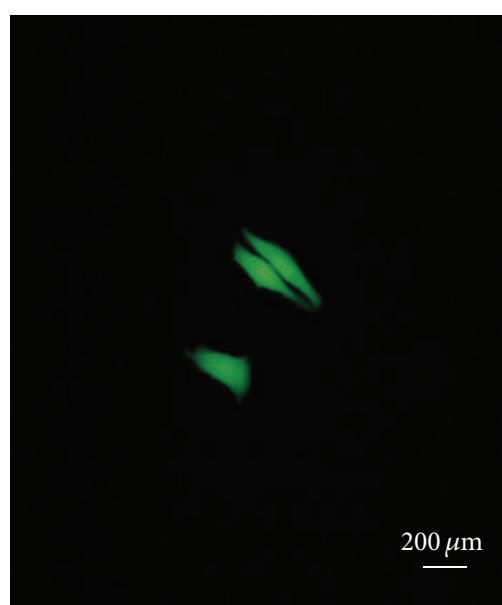

(d)

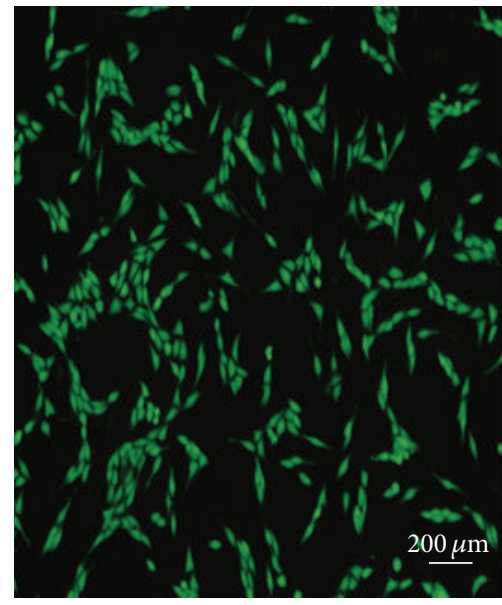

(b)

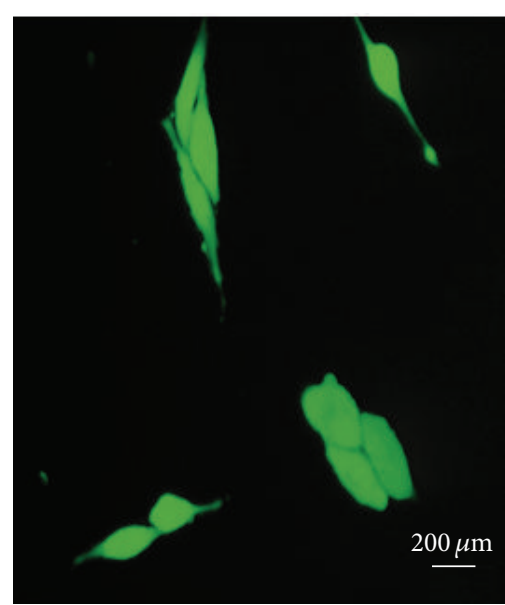

(e)

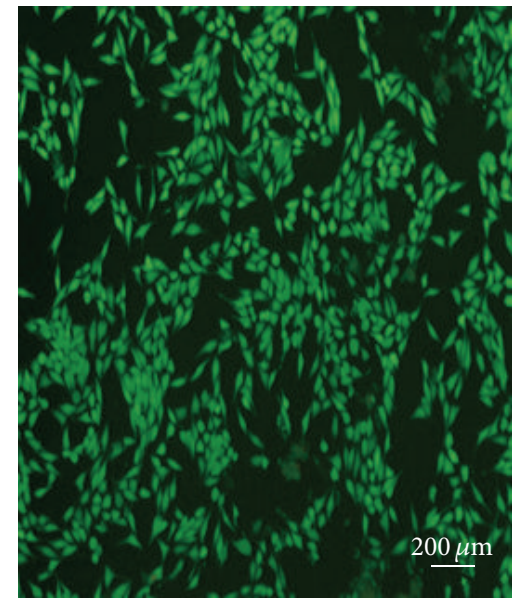

(c)

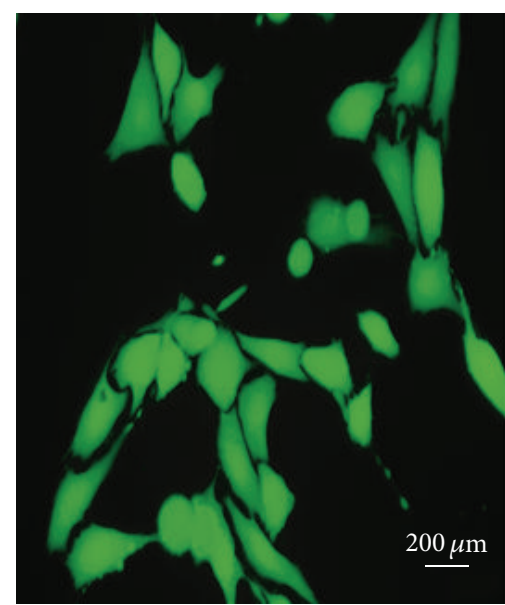

(f)

FIGURE 9: Viability assays of MG63 cells after different days cultured on PVDF scaffolds: ((a) low and (d) high magnified image) 1 day; ((b) low and (e) high magnified image) 3 days; ((c) low and (f) high magnified image) 5 days. 
PVDF scaffolds fabricated by SLS are potential candidates for bone regeneration.

\section{Conflict of Interests}

The authors declare that there is no conflict of interests regarding the publication of this paper.

\section{Acknowledgments}

This work was supported by the following funds: (1) the Natural Science Foundation of China (51222506, 81372366, and 81472058); (2) High Technology Research and Development Program of China (2015AA033503); (3) Overseas, Hong Kong \& Macao Scholars Collaborated Researching Fund of National Natural Science Foundation of China (81428018); (4) Hunan Provincial Natural Science Foundation of China (14JJ1006); (5) Shenzhen Strategic Emerging Industrial Development Funds (JCYJ20130401160614372); (6) the Open-End Fund for the Valuable and Precision Instruments of Central South University; (7) the faculty research grant of Central South University (2013JSJJ011, 2013JSJJ046); (8) State Key Laboratory of New Ceramic and Fine Processing Tsinghua University (KF201413).

\section{References}

[1] G. Zimmermann and A. Moghaddam, "Allograft bone matrix versus synthetic bone graft substitutes," Injury, vol. 42, no. 2, pp. S16-S21, 2011.

[2] T. J. Blokhuis and J. J. C. Arts, "Bioactive and osteoinductive bone graft substitutes: definitions, facts and myths," Injury, vol. 42, no. 2, pp. S26-S29, 2011.

[3] M. Jäger, M. Herten, U. Fochtmann et al., "Bridging the gap: bone marrow aspiration concentrate reduces autologous bone grafting in osseous defects," Journal of Orthopaedic Research, vol. 29, no. 2, pp. 173-180, 2011.

[4] B. Duan, M. Wang, W. Y. Zhou, W. L. Cheung, Z. Y. Li, and W. W. Lu, "Three-dimensional nanocomposite scaffolds fabricated via selective laser sintering for bone tissue engineering," Acta Biomaterialia, vol. 6, no. 12, pp. 4495-4505, 2010.

[5] L. Jin, Z.-Q. Feng, M.-L. Zhu, T. Wang, M. K. Leach, and Q. Jiang, "A novel fluffy conductive polypyrrole nano-layer coated PLLA fibrous scaffold for nerve tissue engineering," Journal of Biomedical Nanotechnology, vol. 8, no. 5, pp. 779-785, 2012.

[6] C. K. Lim, A. S. Halim, I. Zainol, and K. Noorsal, "In vitro evaluation of a biomedical-grade bilayer chitosan porous skin regenerating template as a potential dermal scaffold in skin tissue engineering," International Journal of Polymer Science, vol. 2011, Article ID 645820, 7 pages, 2011.

[7] B. Dhandayuthapani, Y. Yoshida, T. Maekawa, and D. S. Kumar, "Polymeric scaffolds in tissue engineering application: a review," International Journal of Polymer Science, vol. 2011, Article ID 290602, 19 pages, 2011.

[8] J. S. Lee, H. D. Cha, J. H. Shim, J. W. Jung, J. Y. Kim, and D.-W. Cho, "Effect of pore architecture and stacking direction on mechanical properties of solid freeform fabrication-based scaffold for bone tissue engineering," Journal of Biomedical Materials Research: Part A, vol. 100, no. 7, pp. 1846-1853, 2012.
[9] M. Sarem, F. Moztarzadeh, M. Mozafari, and V. P. Shastri, "Optimization strategies on the structural modeling of gelatin/chitosan scaffolds to mimic human meniscus tissue," Materials Science and Engineering C, vol. 33, no. 8, pp. 47774785, 2013

[10] S. Eshraghi and S. Das, "Mechanical and microstructural properties of polycaprolactone scaffolds with one-dimensional, two-dimensional, and three-dimensional orthogonally oriented porous architectures produced by selective laser sintering," Acta Biomaterialia, vol. 6, no. 7, pp. 2467-2476, 2010.

[11] J. M. Hong, B. J. Kim, J.-H. Shim et al., "Enhancement of bone regeneration through facile surface functionalization of solid freeform fabrication-based three-dimensional scaffolds using mussel adhesive proteins," Acta Biomaterialia, vol. 8, no. 7, pp. 2578-2586, 2012.

[12] Y.-J. Seol, T.-Y. Kang, and D.-W. Cho, "Solid freeform fabrication technology applied to tissue engineering with various biomaterials," Soft Matter, vol. 8, no. 6, pp. 1730-1735, 2012.

[13] J.-S. Lee, H. D. Cha, J.-H. Shim, J. W. Jung, J. Y. Kim, and D.-W. Cho, "Effect of pore architecture and stacking direction on mechanical properties of solid freeform fabrication-based scaffold for bone tissue engineering," Journal of Biomedical Materials Research, Part A, vol. 100, no. 7, pp. 1846-1853, 2012.

[14] D. Y. Kwon, J. S. Kwon, S. W. Shim et al., "Preparation of threedimensional scaffolds by using solid freeform fabrication and feasibility study of the scaffolds," Journal of Materials Chemistry B, vol. 2, no. 12, pp. 1689-1698, 2014.

[15] C. P. Jiang, Y. Y. Chen, M. F. Hsieh, and H. M. Lee, "Solid freeform fabrication and in-vitro response of osteoblast cells of mPEG-PCL-mPEG bone scaffolds," Biomedical Microdevices, vol. 15, no. 2, pp. 369-379, 2013.

[16] S. Bose, M. Roy, and A. Bandyopadhyay, "Recent advances in bone tissue engineering scaffolds," Trends in Biotechnology, vol. 30, no. 10, pp. 546-554, 2012.

[17] W. Y. Yeong, N. Sudarmadji, H. Y. Yu et al., "Porous polycaprolactone scaffold for cardiac tissue engineering fabricated by selective laser sintering," Acta Biomaterialia, vol. 6, no. 6, pp. 2028-2034, 2010.

[18] B. Duan, W. L. Cheung, and M. Wang, "Optimized fabrication of Ca-P/PHBV nanocomposite scaffolds via selective laser sintering for bone tissue engineering," Biofabrication, vol. 3, no. 1, Article ID 015001, 2011.

[19] Q. Zhang, X. Lu, and L. Zhao, "Preparation of polyvinylidene fluoride (PVDF) hollow fiber hemodialysis membranes," Membranes, vol. 4, no. 1, pp. 81-95, 2014.

[20] F. O. Agyemang, F. A. Sheikh, R. Appiah-Ntiamoah, J. Chandradass, and H. Kim, "Synthesis and characterization of poly (vinylidene fluoride)-calcium phosphate composite for potential tissue engineering applications," Ceramics International, vol. 41, no. 5, pp. 7066-7072, 2015.

[21] H. F. Guo, Z. S. Li, S. W. Dong et al., "Piezoelectric PU/PVDF electrospun scaffolds for wound healing applications," Colloids and Surfaces B: Biointerfaces, vol. 96, pp. 29-36, 2012.

[22] C. Shuai, C. Gao, Y. Nie, H. Hu, Y. Zhou, and S. Peng, "Structure and properties of nano-hydroxypatite scaffolds for bone tissue engineering with a selective laser sintering system," Nanotechnology, vol. 22, no. 28, Article ID 285703, 2011.

[23] C. Shuai, C. Gao, Y. Nie, A. Hu, H. Qu, and S. Peng, "Structural design and experimental analysis of a selective laser sintering system with nano-hydroxyapatite powder," Journal of Biomedical Nanotechnology, vol. 6, no. 4, pp. 370-374, 2010. 
[24] G. V. Salmoria, P. Klauss, R. A. Paggi, L. A. Kanis, and A. Lago, "Structure and mechanical properties of cellulose based scaffolds fabricated by selective laser sintering," Polymer Testing, vol. 28, no. 6, pp. 648-652, 2009.

[25] A. Pittrof, S. Bauer, and P. Schmuki, "Micropatterned $\mathrm{TiO}_{2}$ nanotube surfaces for site-selective nucleation of hydroxyapatite from simulated body fluid," Acta Biomaterialia, vol. 7, no. 1, pp. 424-431, 2011.

[26] Y. J. Park, Y. S. Kang, and C. Park, "Micropatterning of semicrystalline poly(vinylidene fluoride) (PVDF) solutions," European Polymer Journal, vol. 41, no. 5, pp. 1002-1012, 2005. 

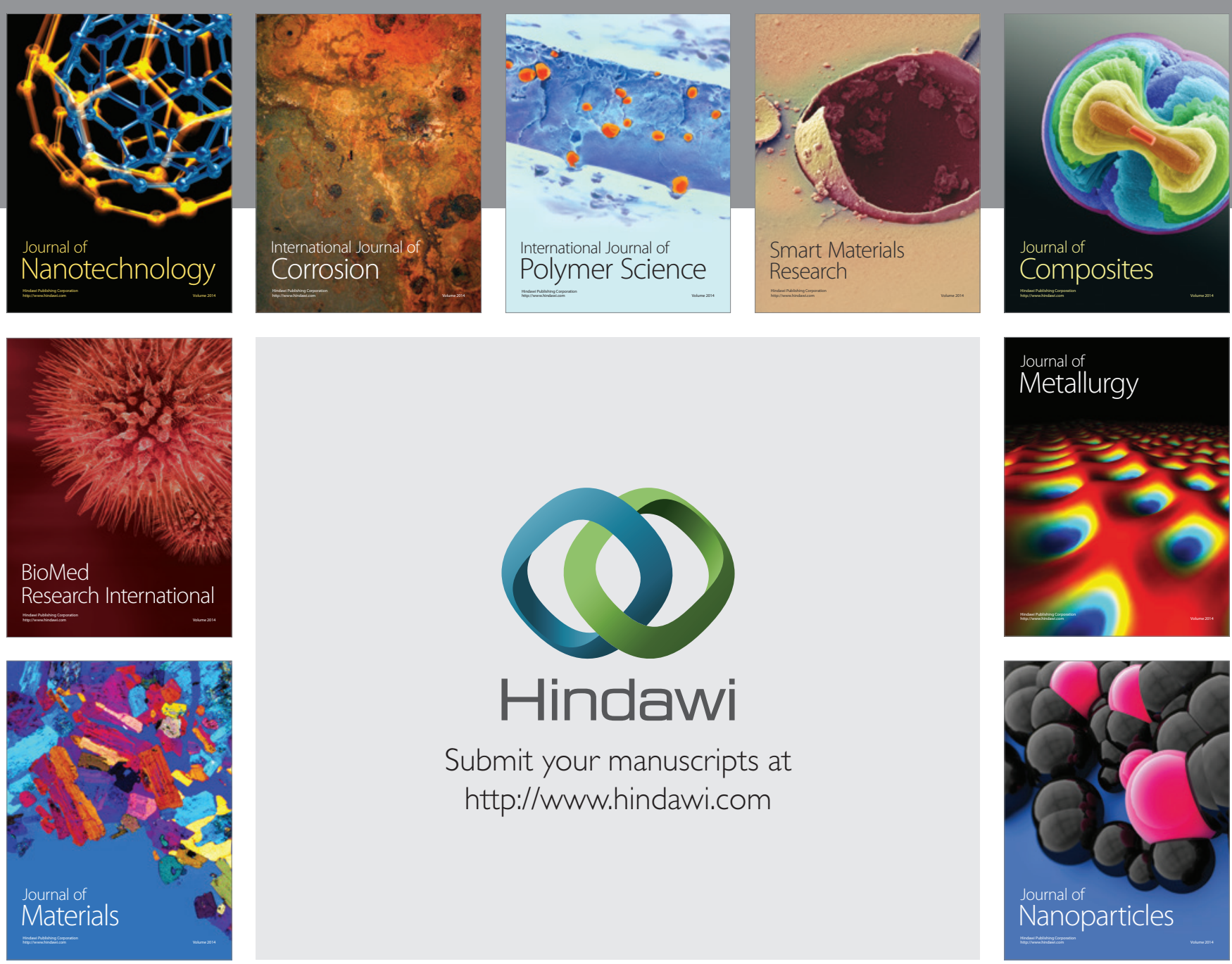

Submit your manuscripts at http://www.hindawi.com
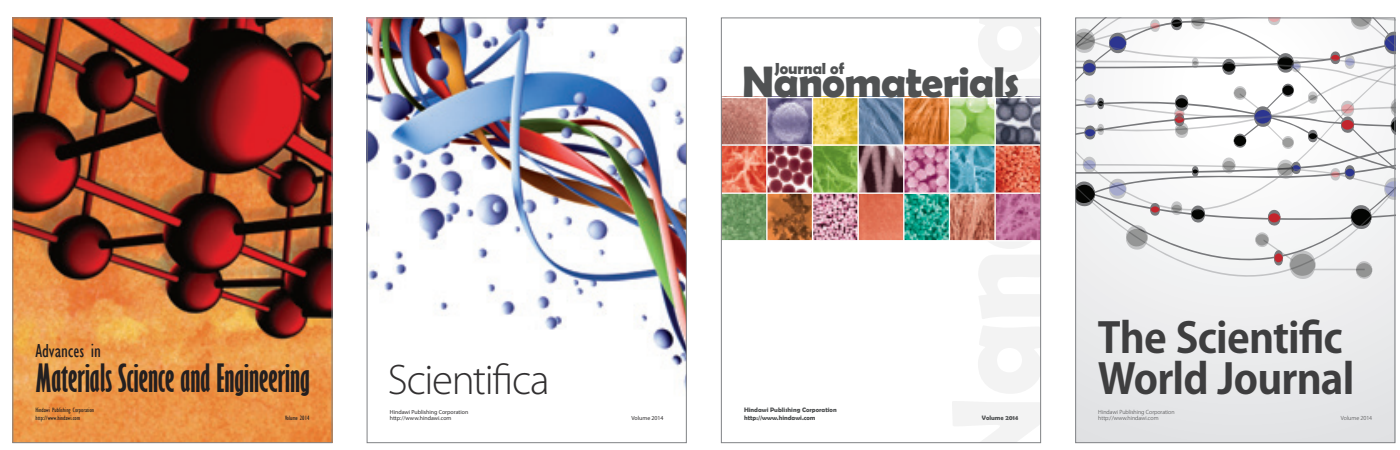

\section{The Scientific World Journal}
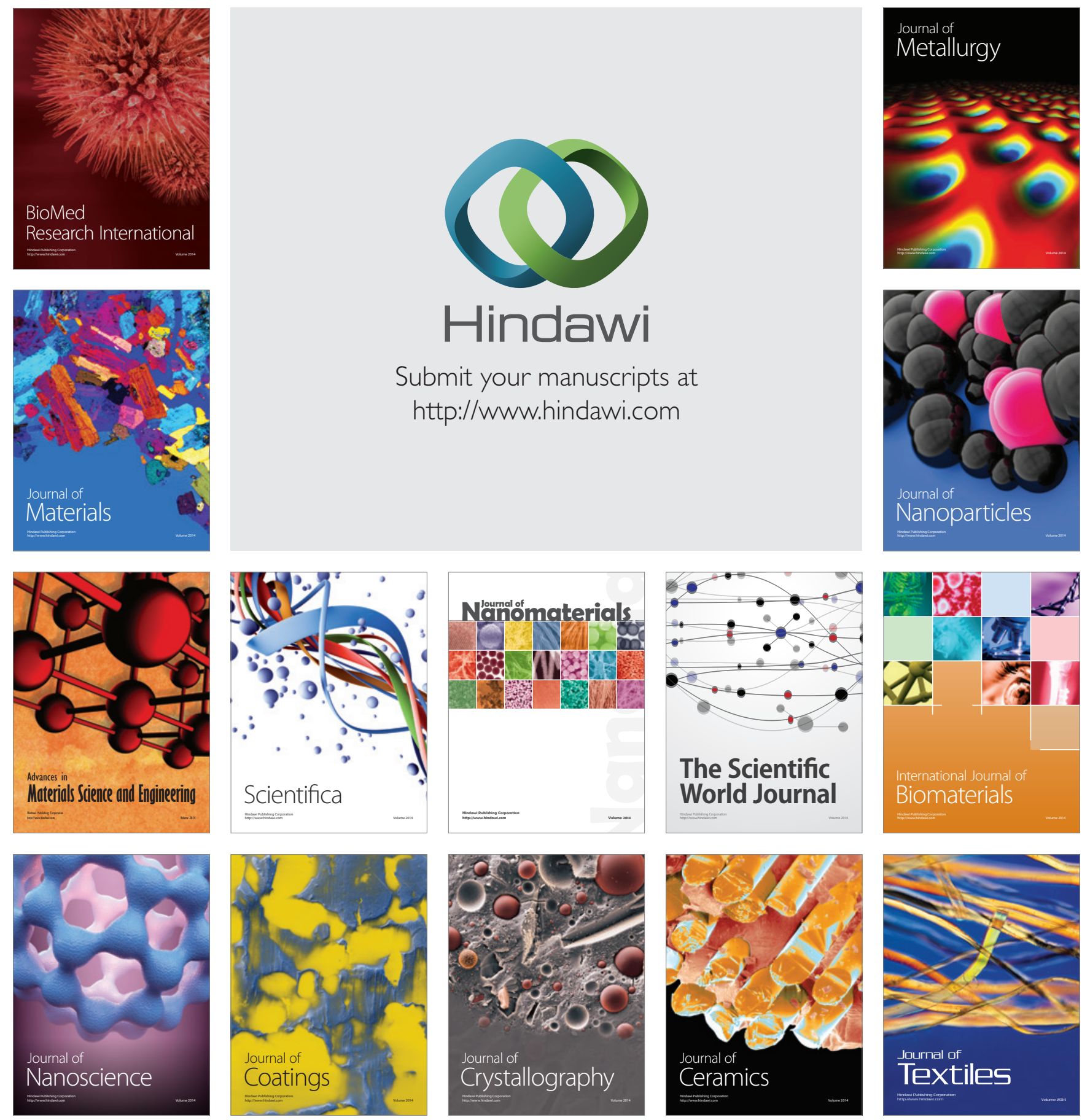